\title{
ERRATUM
}

Volker Koch • Dirk Andrae

\section{Discrete contributions to static dipole polarizabilities of excited bound states of non-relativistic hydrogen-like atoms}

Published online: 1 December 2005

(C) Springer-Verlag 2005

Theor Chem Acc (2005) 114: 380-386

DOI 10.1007/s00214-005-0691-7

A few errors have been found in the article above:

1. In the Introduction, the tenth line should read as: gives another expression for $\varepsilon_{i}^{(2)}$. Restriction to the associated ...

2. The third line following Eq. (2) should read as: solving the unperturbed problem $\left(\widehat{H}_{0}-E_{i}^{(0)}\right) \psi_{i}^{(0)}=0$ (the ...

3. The previously published version of Figure 1 has an incomplete frame of coordinate axes and is blurred. A complete version of Figure 1 is shown below.

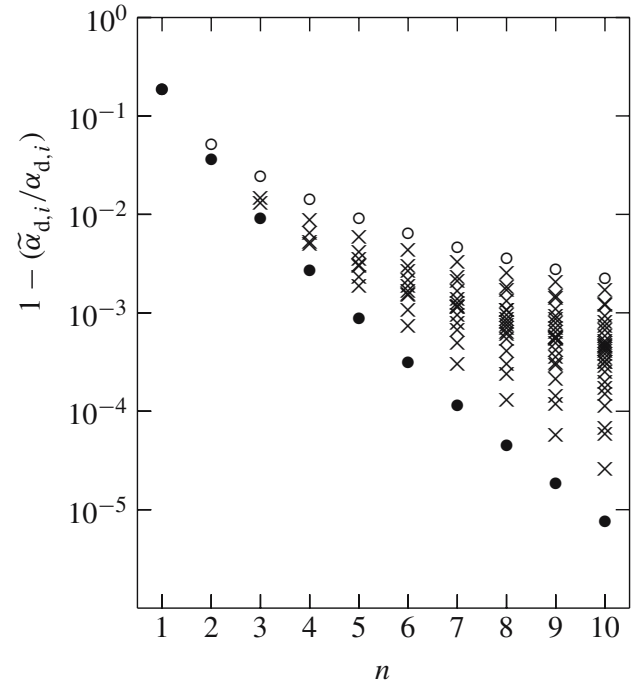

Fig. 1 The relative error of the partial sum $\widetilde{\alpha}_{\mathrm{d}, i}$, Eq. (15) with $\Delta n=64$, for all states $i=(n, q, m)$ with $1 \leq n \leq 10$. The number of states for given $n$ is $N_{\alpha}(n)$, Eq. (13). Filled circles represent data for states $i=(n, 0, n-1)$, cf. Table 1 . Open circles represent data for states $i=(n, n-1,0)$, cf. Table 2 . Crosses represent data for all remaining cases

The online version of the original article can be found at http://dx.doi.org/10.1007/s00214-005-0691-7

V. Koch · D. Andrae ( $\varangle)$

Theoretische Chemie, Fakultät für Chemie, Universität Bielefeld, Postfach 1001 31, D-33501 Bielefeld, Germany,

E-mail: \{v.koch,dirk.andrae\}@uni-bielefeld.de 